\title{
BIODIVERSITY RECORDING AT ROYAL BOTANIC GARDEN EDINBURGH
}

\author{
Robert R. Mill
}

\section{ABSTRACT}

The results of recording wildlife at the Edinburgh site of the Royal Botanic Garden Edinburgh (RBGE) are presented in partial fulfilment of the Garden's obligation to deliver the Scottish Biodiversity Duty. So far, 383 animal species (94 birds, 6 mammals, 3 amphibians, 15 butterflies, 79 moths, 48 hymenopterans, 52 hoverflies, 7 dragonflies and damselflies, 48 other insects, 5 spiders, 8 gall-forming mites, 12 rotifers, 5 cladocerans and 1 copepod) have been recorded. Tables giving lists of the various animal groups are presented. Half of the 103 vertebrates are listed in national Red Lists, the Scottish Biodiversity List or the Edinburgh Local Biodiversity Action Plan List of Notable Species.

\section{INTRODUCTION}

The Royal Botanic Garden Edinburgh has a major international reputation in plant biodiversity research. It is only in recent years, however, that the importance of its four gardens at Edinburgh, Benmore (Argyll), Dawyck (Peeblesshire) and Logan (Dumfries \& Galloway) as reservoirs of biodiversity in their own right has been appreciated.

In addition, as a public body, RBGE now has legal responsibilities and obligations that it previously did not, with respect to biodiversity conservation. Section 1(1) of the Nature Conservation (Scotland) Act (2004), which came into effect on 29 November 2004, states that "It is the duty of every public body and office-holder, in exercising any functions, to further the conservation of biodiversity so far as is consistent with the proper exercise of those functions", while Section 1(2) states that, in complying with the Act, public bodies must have regard to the Scottish Biodiversity Strategy. This was prepared for the Scottish Government by a partnership of public bodies and NGOs known as the Scottish Biodiversity Forum (Scottish Parliament, 2004; Scottish Biodiversity Forum, 2004). More recently, many provisions of the Wildlife and Natural Environment (Scotland) Act 2011 came into force on 1 January 2012, including Section 36 which stipulates that public bodies must prepare and publish a biodiversity report within three years of the base date (Scottish Parliament, 2011a, 2011b).

RBGE also recently began implementing an Environmental Management System (EMS) with a view to receiving accreditation under the international standard ISO 14001.

\footnotetext{
1. Robert Mill is Gymnosperm Systematist at the Royal Botanic Garden Edinburgh and Chairman of the Biodiversity Group there.

Address: 20A Inverleith Row, Edinburgh EH3 5LR.

Email: r.mill@rbge.ac.uk
} 
As part of this EMS, five Environmental Working Groups were set up covering aspects of RBGE's impact on the environment (known as Utilities, Transport, Procurement, Waste and Biodiversity Groups). The Biodiversity Working Group presently comprises eight RBGE staff and volunteers. RBGE has also prepared an Environmental Policy, which states that we will "enhance our role in promoting and protecting biodiversity both locally through the management of our sites in Scotland and internationally through our conservation work in the UK and worldwide" (RBGE, 2011).

Before any public body can begin to protect and conserve the wildlife on its land in order to meet its obligations under the biodiversity legislation outlined above, it needs to know what is there. However, little has been known hitherto about the extent or variety of animal biodiversity that uses the Garden's habitats. The Edinburgh Garden is visited by many species of birds and mammals to feed, drink and bathe, and a large proportion are resident on the site. From spring to the autumn frosts, the Garden is also visited by insects and invertebrates. Many are seeking pollen or nectar and thus benefit the Garden by effecting pollination; others are predators that keep down pests.

\section{METHODS}

Since 2004, I have been recording and monitoring major wildlife groups represented at the Edinburgh Garden. Bird records, in fact, go back to 1997 but more systematic, computerised recording commenced in 2004. Recording of butterflies and terrestrial mammals began in 2007, Odonata (dragonflies and damselflies) in 2008, Hymenoptera (bees, wasps and ants) and hoverflies in 2009, and spiders and leaf-mining insects and mites in 2011. As well as my own records, I have received some for butterflies and Odonata from 2005 onwards from two local naturalists. There have been three efforts to trap moths, and zooplankton were sampled by an external expert in 2010. Garden staff regularly pass on their own sightings to me. There is also a list of bryophytes found in the Scottish Heath Garden; however, that is outside the scope of this paper.

I record wildlife at RBGE each working day throughout the year, which gives a maximum of about 220 possible recording days per year. Recording is done while walking across the Garden to my office in the morning, and during the lunch hour. Birds tend to be most active in the morning and invertebrates during the middle of the day so these two recording periods, though both necessarily time-limited, complement each other well. Field records are, especially in the case of creatures requiring identification, supplemented with digital images taken using the macro facility of a Panasonic TZ6 camera. Records are archived on RBGE's computer network, with separate sets of files for birds, mammals and invertebrates. The day's sightings are also emailed to the John Hope Gateway, where they are put on public display together with a selection of recent wildlife images taken in the Garden.

For each animal group, the following types of record are kept:

- Year lists of species seen, for the calendar year and in the case of birds also for 
the Garden's reporting year (April to March). Since insects are virtually absent between November and February the calendar and reporting year lists would be almost identical, so only the former is kept.

- A cumulative list of all species seen since recording of the group began.

- Since 2007, a monthly list giving the bird sightings for each day; since 2012 this has been extended to include Hymenoptera, hoverflies and butterflies. Also since 2012, the charts on which these monthly data are recorded have been modified to include the Biological Standard Weeks, according to the perpetual system adopted by many biological recording institutions in which each calendar day is always in the same week whatever the year. In this system, week 1 begins on 1 January, week 9 includes 29 February if a leap year, and 31 December is included as an extra day in week 52. Examples of this system can be found in Plant (1994) and Somerset Moth Group (2012).

As part of the Scottish Biodiversity Strategy, a list of species (The Scottish Biodiversity List) has been compiled "that Scottish Ministers consider to be of principal importance for biodiversity conservation in Scotland" (Scottish Natural Heritage, 2010). This list contains 20 terrestrial mammals, 3 reptiles and amphibians (referred to therein as "herptiles"), 93 birds, 289 terrestrial invertebrates, 10 fish, 236 vascular plants, 208 bryophytes, 8 charophytes, 176 fungi, 523 lichens and 240 desmids (Blake, 2005). In addition, a list of "notable species" for Edinburgh has been produced as part of the Edinburgh Local Biodiversity Action Plan (LBAP) (Edinburgh Biodiversity Partnership, 2010a, 2010b). Also, for the animal groups currently recorded at RBGE, there are national Red Lists or equivalent for birds (British Trust for Ornithology, 2009), mammals (Harris et al., 1995) and butterflies (Fox et al., 2010). As well as simply recording species, therefore, those occurring at RBGE are where possible categorised according to whether they are on one or more of these lists. A draft list of notable species occurring at the Edinburgh Garden and at Benmore has recently been compiled, with recommendations on how Garden land can be managed to protect or increase these species where appropriate (Mill, 2012).

\section{RESULTS AND DISCUSSION}

So far (to 30 March 2012), 383 animal species have been recorded at the Edinburgh Garden: 103 vertebrates (94 birds, 6 mammals, 3 amphibians) and 280 invertebrates (15 butterflies, 79 moths, 48 hymenopterans, 52 hoverflies, 7 dragonflies and damselflies, 48 other insects, 5 spiders, 8 gall-forming mites, 12 rotifers, 5 cladocerans and 1 copepod). Where a species has both a scientific and a common name, the latter, e.g. house sparrow, is used in the text, with the scientific name (in that example, Passer domesticus) listed in the relevant table. 


\section{Birds (1997-2012: Table 1)}

Of the 94 bird species, 86 have been recorded since 1997; 43 of those (50 per cent) are either Red- or Amber-listed in Birds of Conservation 3 (British Trust for Ornithology, 2009) with 11 (plus one now locally extinct) Red-Listed. Three Red-Listed species (herring gull, song thrush, house sparrow) are resident or daily visitors although house sparrows are now confined to two small roost sites, one of which has apparently been unused for almost a year. Two (redwing and fieldfare) are winter visitors, while six (Arctic skua, lesser redpoll, linnet, skylark, spotted flycatcher and starling) are only occasional. Spotted flycatcher, however, bred in the Garden at least until the 1980s (McBeath, 1989). Hawfinch, another Red-listed bird, also frequented the Edinburgh Garden from 1947 and annually between 1974 and 1992 (McBeath, 1989; Duncan, 1999; H. Noltie, pers. comm.) but is now absent throughout Edinburgh apart from very occasional unconfirmed sightings. The 31 Amber-Listed species include 5 resident breeders (bullfinch, dunnock, mallard, mistle thrush, stock dove), 3 gull species that visit regularly, 4 summer visitors (house martin, swallow, swift, willow warbler), 4 other regular visitors (grey wagtail, kingfisher, mute swan, oystercatcher; swans have attempted to breed, so far unsuccessfully) and 15 occasional visitors or passage migrants.

Less common breeding birds include sparrowhawk (monitored by Lothian and Borders Raptor Study Group who have also collaborated with RBGE to get webcam footage of the nests to the John Hope Gateway), great spotted woodpecker and, at least until recently, tawny owl, which seems to have become much less regular in its visits to RBGE in the last few years.

Unusual bird visitors to the Edinburgh Garden have included crossbill in July 2008 (a national irruption year for the species) and winter 2010, no fewer than five goosanders on the Pond on 18 October 2011, four grey plovers bathing in the recently created Biodiversity Garden ponds (10 November 2011), peregrine, pheasant, raven, reed bunting (a singing male, 2006), sedge warbler (another singing male, 2007), skylark (on a very snowy day in December 2010), a snipe with a damaged bill that had to be humanely despatched by the RSPB and woodcock (late autumn/winter records in 2007, 2010 and 2011). The most extraordinary bird to have visited the Garden so far must surely be a white stork that turned up on 22 May 2011 and later that week visited the Gyle shopping centre and Princes Street Gardens.

Daily recording of birds has resulted in the discovery that RBGE is on a migration route for meadow pipit, visible migration of which can often be seen for a few days in spring (migrating inland) and autumn (migrating east, out to sea). Some species have dramatically increased, such as goldfinch, while others have greatly declined in frequency of occurrence or have disappeared completely, such as collared dove. Table 1 includes eight species that used to occur at least occasionally in RBGE or the skies above prior to 1990 (McBeath, 1989) but have not been seen since my recording began. 


\begin{tabular}{|c|c|c|c|c|c|}
\hline & Common name & Scientific name & Family & Listings & Years recorded \\
\hline 1 & Sparrowhawk & Accipiter nisus & Accipitridae & $\mathrm{E}$ & $00-12$ \\
\hline 2 & Buzzard & Buteo buteo & Accipitridae & $\mathrm{E}$ & $02,06-12$ \\
\hline 3 & Long-tailed tit & Aegithalos caudatus & Aegithalidae & & $97-12$ \\
\hline 4 & Skylark & Alauda arvensis & Alaudidae & $\mathrm{R}, \mathrm{S}, \mathrm{E}$ & 10 \\
\hline 5 & Kingfisher & Alcedo atthis & Alcedinidae & $\mathrm{A}, \mathrm{S}, \mathrm{E}$ & $98-02,04-12$ \\
\hline 6 & Little auk & Alle alle & Alcidae & & $\mathrm{H}$ \\
\hline 7 & Wigeon & Anas penelope & Anatidae & $\mathrm{A}, \mathrm{E}$ & $\mathrm{H}$ \\
\hline 8 & Mallard & Anas platyrhynchos & Anatidae & $\mathrm{A}, \mathrm{E}$ & $97-12$ \\
\hline 9 & Greylag goose & Anser anser & Anatidae & $\mathrm{A}, \mathrm{E}$ & $08-11$ \\
\hline 10 & Pink-footed goose & Anser brachyrhynchus & Anatidae & $\mathrm{A}, \mathrm{E}$ & $98-99,01,04-12$ \\
\hline 11 & Tufted duck & Aythya fuligula & Anatidae & A & $00,03,06,10,11$ \\
\hline 12 & Canada goose & Branta canadensis & Anatidae & & 09 \\
\hline 13 & Goldeneye & Bucephala clangula & Anatidae & $\mathrm{A}, \mathrm{E}$ & 09 \\
\hline 14 & Whooper swan & Cygnus cygnus & Anatidae & A, S, E & 06,10 \\
\hline 15 & Mute swan & Cygnus olor & Anatidae & $\mathrm{A}, \mathrm{E}$ & $00-12$ \\
\hline 16 & Goosander & Mergus merganser & Anatidae & $\mathrm{E}$ & 11 \\
\hline 17 & Swift & Apus apus & Apodidae & A, S, E & $00-12$ \\
\hline 18 & Grey heron & Ardea cinerea & Ardeidae & & $97-12$ \\
\hline 19 & Night heron & Nycticorax nycticorax & Ardeidae & & $\mathrm{H}$ \\
\hline 20 & Waxwing & Bombycilla garrulus & Bombycillidae & & $00-05,08,10,11$ \\
\hline 21 & Treecreeper & Certhia familiaris & Certhiidae & & $97-12$ \\
\hline 22 & Grey plover & Pluvialis squatarola & Charadriidae & A & 11 \\
\hline 23 & White stork & Ciconia ciconia & Ciconiidae & & 11 \\
\hline 24 & Dipper & Cinclus cinclus & Cinclidae & & $\mathrm{H}$ \\
\hline 25 & Feral pigeon (rock dove) & Columba livia & Columbidae & & $97-12$ \\
\hline 26 & Stock dove & Columba oenas & Columbidae & $\mathrm{A}, \mathrm{E}$ & $99-12$ \\
\hline 27 & Woodpigeon & Columba palumbus & Columbidae & & $97-12$ \\
\hline 28 & Collared dove & Streptopelia decaocto & Columbidae & & $97-05,10$ \\
\hline 29 & Raven & Corvus corax & Corvidae & & 09,10 \\
\hline 30 & Hooded crow & Corvus cornix & Corvidae & $\mathrm{S}$ & 08,11 \\
\hline 31 & Carrion crow & Corvus corone & Corvidae & & $97-12$ \\
\hline 32 & Rook & Corvus frugilegus & Corvidae & & $04-06,11$ \\
\hline 33 & Jackdaw & Corvus monedula & Corvidae & & $08-12$ \\
\hline 34 & Jay & Garrulus glandarius & Corvidae & & 11 \\
\hline
\end{tabular}




\begin{tabular}{|c|c|c|c|c|c|}
\hline & Common name & Scientific name & Family & Listings & Years recorded \\
\hline 35 & Magpie & Pica pica & Corvidae & & $97-12$ \\
\hline 36 & Reed bunting & Emberiza schoeniclus & Emberizidae & $\mathrm{A}, \mathrm{S}, \mathrm{E}$ & 06 \\
\hline 37 & Peregrine & Falco peregrinus & Falconidae & $\mathrm{S}, \mathrm{E}$ & 08,09 \\
\hline 38 & Kestrel & Falco tinnunculus & Falconidae & $\mathrm{A}, \mathrm{S}, \mathrm{E}$ & 07,12 \\
\hline 39 & Lesser redpoll & Carduelis cabaret & Fringillidae & $\mathrm{R}$ & 09,10 \\
\hline 40 & Linnet & Carduelis cannabina & Fringillidae & $\mathrm{R}, \mathrm{S}, \mathrm{E}$ & $\begin{array}{l}97,98,01,04-08, \\
10\end{array}$ \\
\hline 41 & Goldfinch & Carduelis carduelis & Fringillidae & & $98,01,02,04-12$ \\
\hline 42 & Siskin & Carduelis spinus & Fringillidae & $S$ & $98-12$ \\
\hline 43 & Greenfinch & Chloris chloris & Fringillidae & & $98-12$ \\
\hline 44 & Hawfinch & $\begin{array}{l}\text { Coccothraustes } \\
\text { coccothraustes }\end{array}$ & Fringillidae & $\mathrm{R}, \mathrm{S}$ & $\begin{array}{l}\text { pre-97; } \\
\text { unconfirmed more } \\
\text { recent sightings }\end{array}$ \\
\hline 45 & Chaffinch & Fringilla coelebs & Fringillidae & & $97-12$ \\
\hline 46 & Brambling & Fringilla montifringilla & Fringillidae & $\mathrm{S}, \mathrm{E}$ & 11 \\
\hline 47 & Crossbill & Loxia curvirostra & Fringillidae & & 08,10 \\
\hline 48 & Bullfinch & Pyrrhula pyrrhula & Fringillidae & $\mathrm{A}, \mathrm{S}, \mathrm{E}$ & $98-12$ \\
\hline 49 & Oystercatcher & Haematopus ostralegus & Haematopodidae & $\mathrm{A}, \mathrm{E}$ & $02-12$ \\
\hline 50 & House martin & Delichon urbica & Hirundinidae & $\mathrm{A}, \mathrm{E}$ & $98,00-11$ \\
\hline 51 & Swallow & Hirundo rustica & Hirundinidae & $\mathrm{A}, \mathrm{E}$ & $04-11$ \\
\hline 52 & Blackheaded gull & $\begin{array}{l}\text { Chroicocephalus } \\
\text { ridibundus }\end{array}$ & Laridae & A, S & $98,02-12$ \\
\hline 53 & Herring gull & Larus argentatus & Laridae & $\mathrm{R}, \mathrm{S}, \mathrm{E}$ & $98-12$ \\
\hline 54 & Common (mew) gull & Larus canus & Laridae & $\mathrm{A}, \mathrm{E}$ & $03-11$ \\
\hline 55 & $\begin{array}{l}\text { Lesser black-backed } \\
\text { gull }\end{array}$ & Larus fuscus & Laridae & $\mathrm{A}, \mathrm{E}$ & $98,01-12$ \\
\hline 56 & Meadow pipit & Anthus pratensis & Motacillidae & $\mathrm{A}, \mathrm{E}$ & $07-09,11$ \\
\hline 57 & Pied wagtail & Motacilla alba & Motacillidae & & $00,02-11$ \\
\hline 58 & Grey wagtail & Motacilla cinerea & Motacillidae & A & $05-11$ \\
\hline 59 & Spotted flycatcher & Muscicapa striata & Muscicapidae & $\mathrm{R}, \mathrm{S}, \mathrm{E}$ & 09 \\
\hline 60 & Blue tit & Cyanistes caeruleus & Paridae & & $97-12$ \\
\hline 61 & Great tit & Parus major & Paridae & & $97-12$ \\
\hline 62 & Coal tit & Periparus ater & Paridae & & $97-12$ \\
\hline 63 & House sparrow & Passer domesticus & Passeridae & $\mathrm{R}, \mathrm{E}$ & $03,06-11$ \\
\hline 64 & Cormorant & Phalacrocorax carbo & Phalacrocoridae & $\mathrm{E}$ & $98,02,07,10,12$ \\
\hline 65 & Pheasant & Phasianus colchicus & Phasianidae & & $\begin{array}{l}97,99,05-07, \\
11-12\end{array}$ \\
\hline
\end{tabular}




\begin{tabular}{|c|c|c|c|c|c|}
\hline & Common name & Scientific name & Family & Listings & Years recorded \\
\hline 66 & $\begin{array}{l}\text { Great spotted } \\
\text { woodpecker }\end{array}$ & Dendrocopos major & Picidae & & $01,03-12$ \\
\hline 67 & Green woodpecker & Picus viridis & Picidae & $\mathrm{A}, \mathrm{E}$ & 02,09 \\
\hline 68 & Dabchick (little grebe) & Tachybaptus ruficollis & Podicipedidae & A & 01,08 \\
\hline 69 & Fulmar & Fulmarus glacialis & Procellaridae & $\mathrm{A}, \mathrm{E}$ & $\mathrm{H}$ \\
\hline 70 & Dunnock & Prunella modularis & Prunellidae & $\mathrm{A}, \mathrm{E}$ & $97-12$ \\
\hline 71 & Coot & Fulica atra & Rallidae & & $\mathrm{H}$ \\
\hline 72 & Moorhen & Gallinula chloropus & Rallidae & & $97-12$ \\
\hline 73 & Water rail & Rallus aquaticus & Rallidae & $\mathrm{E}$ & $\mathrm{H}$ \\
\hline 74 & Snipe & Gallinago gallinago & Scolopacidae & $\mathrm{A}, \mathrm{E}$ & 09 \\
\hline 75 & Curlew & Numenius arquata & Scolopacidae & $\mathrm{A}, \mathrm{S}, \mathrm{E}$ & 04-12 \\
\hline 76 & Woodcock & Scolopax rusticola & Scolopacidae & $\mathrm{A}, \mathrm{S}, \mathrm{E}$ & $07,10,11$ \\
\hline 77 & Redshank & Tringa totanus & Scolopacidae & $\mathrm{A}, \mathrm{E}$ & $04,09-11$ \\
\hline 78 & Arctic skua & Stercorarius parasiticus & Stercorariidae & $\mathrm{R}, \mathrm{S}$ & 09 \\
\hline 79 & Short-eared owl & Asio flammeus & Strigidae & A, S & $\mathrm{H}$ \\
\hline 80 & Tawny owl & Strix aluco & Strigidae & & $97-08,10,11$ \\
\hline 81 & Starling & Sturnus vulgaris & Sturnidae & $\mathrm{R}, \mathrm{E}$ & $01,04,05$ \\
\hline 82 & Sedge warbler & $\begin{array}{l}\text { Acrocephalus } \\
\text { schoenobaenus }\end{array}$ & Sylviidae & & 07 \\
\hline 83 & Chiffchaff & Phylloscopus collybita & Sylviidae & & $98,00,02-12$ \\
\hline 84 & Willow warbler & Phylloscopus trochilus & Sylviidae & $\mathrm{A}, \mathrm{E}$ & $97-11$ \\
\hline 85 & Goldcrest & Regulus regulus & Sylviidae & $\mathrm{E}$ & $97-12$ \\
\hline 86 & Blackcap & Sylvia atricapilla & Sylviidae & & $00,03-12$ \\
\hline 87 & Garden warbler & Sylvia borin & Sylviidae & & 97,12 \\
\hline 88 & Wren & Troglodytes troglodytes & Troglodytidae & & $98-12$ \\
\hline 89 & Robin & Erithacus rubecula & Turdidae & $\mathrm{S}, \mathrm{E}$ & $97-12$ \\
\hline 90 & Redwing & Turdus iliacus & Turdidae & $\mathrm{R}, \mathrm{S}, \mathrm{E}$ & $97,98,00-12$ \\
\hline 91 & Blackbird & Turdus merula & Turdidae & & $97-12$ \\
\hline 92 & Song thrush & Turdus philomelos & Turdidae & $\mathrm{R}, \mathrm{S}, \mathrm{E}$ & $97-12$ \\
\hline 93 & Fieldfare & Turdus pilaris & Turdidae & $\mathrm{R}, \mathrm{E}$ & $02,07,09-11$ \\
\hline 94 & Mistle thrush & Turdus viscivorus & Turdidae & $\mathrm{A}, \mathrm{E}$ & $01-12$ \\
\hline
\end{tabular}

Table 1 Birds recorded at RBGE's Edinburgh Garden, 1997-2012 (March). Listings: R: UK National BAP Red List; A: UK National BAP Amber List; S: Scottish Biodiversity List; E: Edinburgh LBAP Notable Species list. The list also includes eight species not seen since 1997 but listed as occurring at RBGE in the past by McBeath (1989). These are indicated by ' $\mathrm{H}$ ' (historic) in the last column. In all tables the first two digits of the year are omitted, e.g. $02=2002$. 


\section{Mammals and amphibians (2007-2012: Table 2)}

At least two of the six mammals (badger, otter) and all three amphibians (common frog, smooth and palmate newts) so far recorded are on the Scottish Biodiversity List, although the palmate newts were apparently deliberately introduced in 2011 ( $\mathrm{H}$. Coventry, pers. comm.). The only ones that visitors are likely to see, apart from the ever-present grey squirrels, are an occasional daytime-roaming fox, and common frog. Evidence of the nocturnal activities of the badgers that arrived at the Edinburgh Garden in 2008 is, however, all too apparent in their damage to the lawns. Since their arrival the badgers have made numerous setts around the site, all of which have been mapped in order that the Garden can meet its obligations to protect this Lothian LBAP species under the Protection of Badgers Act 1992. An otter, probably disturbed by ongoing flood prevention works at the nearby Water of Leith, was seen in November 2011. Pipistrelle bats visit the Edinburgh Garden at dusk on warm evenings from spring to autumn; it is not yet known if these are common pipistrelles or soprano pipistrelles, the recently segregated species that has a higher-pitched echo-location frequency. Both are Scottish Biodiversity List species. Elsewhere in Edinburgh and Lothians, there is evidence that soprano pipistrelle is the commoner of the two species (Clear, 2005; Middleton, 2006); one cannot exclude the possibility that both species may visit the Garden. Nothing is yet known about any small nocturnal mammals that may frequent the Garden.

\begin{tabular}{|l|l|l|l|l|l|}
\hline & \multicolumn{1}{|c|}{ Common name } & \multicolumn{1}{|c|}{ Scientific name } & \multicolumn{1}{|c|}{ Family } & Listings & \multicolumn{1}{|l|}{ Years recorded } \\
\hline 1 & Fox & Vulpes vulpes & Canidae & & $07-12$ \\
\hline 2 & Field vole & Microtus agrestis & Cricetidae & & 09 \\
\hline 3 & Badger & Meles meles & Mustelidae & S, E & $09-12$ \\
\hline 4 & Otter & Lutra lutra & Mustelidae & E & 11 \\
\hline 5 & Grey squirrel & Sciurus carolinensis & Sciuridae & & $07-12$ \\
\hline 6 & $\begin{array}{l}\text { Common \& soprano } \\
\text { pipistrelle bats }\end{array}$ & $\begin{array}{l}\text { Pipistrellus pipistrellus } \\
\text { [common], P. pygmaeus } \\
{[\text { soprano] }}\end{array}$ & Vespertilionidae & S (both), & 07 \\
\hline 7 & Common frog & Rana temporaria & Ranidae & & $09,11,12$ \\
\hline 8 & Smooth newt & Lissotriton vulgaris & Salamandridae & & 09 \\
\hline 9 & Palmate newt & Lissotriton helveticus & Salamandridae & & 11 \\
\hline
\end{tabular}

Table 2 Mammals and amphibians recorded at RBGE's Edinburgh Garden, 2007-2012. Listings codes as for Table 1 .

\section{Butterflies and moths (2005-2012: Tables 3 and 4)}

Fifteen butterfly species have been recorded at RBGE by me or Hugh and Laura Coventry (pers. comm.), although one (large white) has not been seen for several years (Table 3). None is endangered or on the Scottish Biodiversity or Edinburgh LBAP Lists. 
However, several are species that have recently spread dramatically in Scotland, almost certainly as a result of climate change. These include comma, peacock, speckled wood and holly blue. The spreads or invasions of all these (and others not yet recorded from RBGE) in Scotland have been documented by Sutcliffe (2009). RBGE is notable for having one of the few Scottish colonies of the holly blue, which in a good season can produce two broods, flying in spring (April/May) and late summer (July/August). It was first reported at RBGE in 2007 (Sutcliffe, 2007 and own records) and has been seen annually since, except in 2009 when it was surely overlooked. The comma had been absent from Scotland for 130 years until it was reported in 1996, in the Borders. Since then it has spread rapidly northwards. The first Lothians record was in 2002 (Sutcliffe, 2009); it has been recorded at RBGE since 2008. The first confirmed occurrence of speckled wood in Edinburgh was on 8 June 2008 when one was seen "on a riverside path shaded with trees" (Butterfly Conservation, 2008); there are RBGE records from 13 June 2008 (unconfirmed) and 2011 (H. Coventry, pers. comm.).

A total of 79 moth species have been recorded at RBGE (Table 4). Most were trapped on the evenings of 20 June 2008, 3 and 4 July 2009 and 15 August 2009, with the July 2009 evenings yielding most records. All are common species, not on the Edinburgh LBAP or Scottish Biodiversity Lists. On 21 July 2011, I recorded yarrow plume-moth (Fig. 1) resting on its food plant, yarrow (Achillea millefolium) - surprisingly the first record of this species for Midlothian (v.c. 83) although it is widespread in Scotland.

\begin{tabular}{|c|l|l|l|l|}
\hline \multicolumn{1}{|c|}{ Common name } & \multicolumn{1}{|c|}{ Scientific name } & \multicolumn{1}{c|}{ Family } & \multicolumn{1}{c|}{ Years recorded } \\
\hline 1 & Holly blue & Celastrina argiolus & Lycaenidae & $07,08,10,11$ \\
\hline 2 & Small copper* & Lycaena phlaeas & Lycaenidae & $08-11$ \\
\hline 3 & Common blue* & Polyommatus icarus & Lycaenidae & $08,09,10$ \\
\hline 4 & Orange-tip* & Anthocharis cardamines & Pieridae & 10 \\
\hline 5 & Large white & Pieris brassicae & Pieridae & pre-2008 \\
\hline 6 & Green-veined white* & Pieris napi & Pieridae & $09,10,11$ \\
\hline 7 & Small white* & Pieris rapae & Pieridae & $07-11$ \\
\hline 8 & Ringlet* & Aphantopus hyperantus & Satyridae & $08 *, 10$ \\
\hline 9 & Meadow brown* & Maniola jurtina & Satyridae & $08,09,10$ \\
\hline 10 & Speckled wood & Pararge aegeria & Satyridae & 08 unconfirmed, 11 \\
\hline 11 & Small tortoiseshell* & Aglais urticae & Vanessidae & $07-11$ \\
\hline 12 & Peacock* & Inachis io & Vanessidae & $07-11$ \\
\hline 13 & Comma* & Polygonia c-album & Vanessidae & $08-11$ \\
\hline 14 & Red admiral* & Vanessa atalanta & Vanessidae & $07-11$ \\
\hline 15 & Painted lady* & Vanessa cardui & Vanessidae & $07,08,10$ \\
\hline
\end{tabular}

Table 3 Butterflies recorded at RBGE's Edinburgh Garden, 2007-2011. An asterisk (*) after the English name indicates that the species was also recorded prior to 2007 (H. Coventry, pers. comm.). None is Listed. 


\begin{tabular}{|c|c|c|c|c|}
\hline & Common name & Scientific name & Family & Years recorded \\
\hline 1 & Ghost moth & Hepialus humuli humuli & Hepialidae & 08 \\
\hline 2 & Map-winged swift & Hepialus fusconebulosa & Hepialidae & 09 \\
\hline 3 & a micro-moth & Stigmella splendidissimella & Nepticulidae & 09 \\
\hline 4 & a micro-moth & Stigmella sorbi & Nepticulidae & $11^{*}$ \\
\hline 5 & a micro-moth & Stigmella tityrella & Nepticulidae & 09 \\
\hline 6 & a micro-moth & Incurvaria pectinea & Incurvariidae & $11 *$ \\
\hline 7 & a micro-moth & Heliozela sericiella & Heliozelidae & 09 \\
\hline 8 & Apple leaf miner & Lyonetia clerkella & Lyonetiidae & $09,11 *$ \\
\hline 9 & a micro-moth & Caloptilia rufipennella & Gracillariidae & 09 \\
\hline 10 & a micro-moth & Caloptilia syringella & Gracillariidae & $09,11^{*}$ \\
\hline 11 & a micro-moth & Parornix scoticella & Gracillariidae & $11 *$ \\
\hline 12 & a micro-moth & Phyllonorycter maestingella & Gracillariidae & 09 \\
\hline 13 & a micro-moth & Phyllonorycter ulmifoliella & Gracillariidae & 09 \\
\hline 14 & a micro-moth & Argyresthia brockeella & Yponomeutidae & 09 \\
\hline 15 & a micro-moth & Argyresthia goedartella & Yponomeutidae & 09 \\
\hline 16 & Diamond-back moth & Plutella xylostella & Yponomeutidae & 09 \\
\hline 17 & Brown house-moth & Hofmannophila pseudospretella & Oecophoridae & 09 \\
\hline 18 & a micro-moth & Carcina quercana & Oecophoridae & 09 \\
\hline 19 & a micro-moth & Blastobasis adustella (= lignea) & Blastobasidae & 09 \\
\hline 20 & a micro-moth & Blastobasis decolorella & Blastobasidae & 08 \\
\hline 21 & Dark fruit-tree tortrix & Pandemis heparana & Tortricidae & 09 \\
\hline 22 & a micro-moth & Aphelia unitana & Tortricidae & 09 \\
\hline 23 & Light brown apple moth & Epiphyas postvittana & Tortricidae & $\begin{array}{l}10 \text { (LS pers. } \\
\text { comm.) }\end{array}$ \\
\hline 24 & a micro-moth & Lozotaenia forsterana & Tortricidae & 09 \\
\hline 25 & a micro-moth & Acleris sparsana & Tortricidae & 09 \\
\hline 26 & a micro-moth & Celypha lacunana & Tortricidae & 09 \\
\hline 27 & Nut bud moth & Epinotia tenerana & Tortricidae & 09 \\
\hline 28 & a micro-moth & Lathronympha strigana & Tortricidae & 09 \\
\hline 29 & a micro-moth & Chrysoteuchia culmella & Crambidae & 09 \\
\hline 30 & a micro-moth & Agriphila straminella & Crambidae & 09 \\
\hline 31 & a micro-moth & Agriphila tristella & Crambidae & 09 \\
\hline 32 & a micro-moth & Scoparia ambigualis & Crambidae & 09 \\
\hline 33 & a micro-moth & Udea olivalis & Crambidae & 08,09 \\
\hline
\end{tabular}




\begin{tabular}{|c|c|c|c|c|}
\hline & Common name & Scientific name & Family & Years recorded \\
\hline 34 & Bee moth & Aphomia sociella & Pyralidae & 09 \\
\hline 35 & Beautiful plume & Amblyptilia acanthadactyla & Pterophoridae & 09 \\
\hline 36 & Triangle plume & Platyptilia gonodactyla & Pterophoridae & 09 \\
\hline 37 & Yarrow plume & Platyptilia pallidactyla & Pterophoridae & $11 * \mathrm{NCR}$ \\
\hline 38 & Common lutestring & Ochropacha duplaris & Thyatiridae & 08,09 \\
\hline 39 & Small fan-footed wave & Idaea biselata & Geometridae & 09 \\
\hline 40 & Small dusty wave & Idaea seriata & Geometridae & 09 \\
\hline 41 & Riband wave & Idaea aversata & Geometridae & 09 \\
\hline 42 & Silver-ground carpet & Xanthorhoe montanata & Geometridae & 08,09 \\
\hline 43 & The phoenix & Eulithis prunata & Geometridae & 09 \\
\hline 44 & $\begin{array}{l}\text { Common marbled } \\
\text { carpet }\end{array}$ & Chloroclysta truncata & Geometridae & 08,09 \\
\hline 45 & Barred yellow & Cidaria fulvata & Geometridae & 09 \\
\hline 46 & Spruce carpet & Thera britannica & Geometridae & 08 \\
\hline 47 & Clouded border & Lomaspilis marginata & Geometridae & 09 \\
\hline 48 & Brimstone moth & Opisthograptis luteolata & Geometridae & $08,09,10^{*}$ \\
\hline 49 & Peppered moth & Biston betularia & Geometridae & 09 \\
\hline 50 & Willow beauty & Peribatodes rhomboidaria & Geometridae & 09 \\
\hline 51 & Satin beauty & Deileptenia ribeata & Geometridae & 09 \\
\hline 52 & Mottled beauty & Alcis repandata & Geometridae & 09 \\
\hline 53 & Bordered white & Bupalus piniaria & Geometridae & 09 \\
\hline 54 & Common white wave & Cabera pusaria & Geometridae & 09 \\
\hline 55 & Light emerald & Campaea margaritata & Geometridae & 09 \\
\hline 56 & Barred red & Hylaea fasciaria & Geometridae & 08,09 \\
\hline 57 & Poplar hawkmoth & Laothoe populi & Sphingidae & $11^{*}$ \\
\hline 58 & Turnip moth & Agrotis segetum & Noctuidae & 08 \\
\hline 59 & Heart and dart & Agrotis exclamationis & Noctuidae & 08 \\
\hline 60 & Large yellow underwing & Noctua pronuba & Noctuidae & 08,09 \\
\hline 61 & $\begin{array}{l}\text { Lesser yellow } \\
\text { underwing }\end{array}$ & Noctua comes & Noctuidae & 09 \\
\hline 62 & $\begin{array}{l}\text { Lesser broad-bordered } \\
\text { yellow underwing }\end{array}$ & Noctua janthe & Noctuidae & 09 \\
\hline 63 & Purple clay & Diarsia brunnea & Noctuidae & 09 \\
\hline 64 & Double square-spot & Xestia triangulum & Noctuidae & 09 \\
\hline 65 & Dotted clay & Xestia baja & Noctuidae & 09 \\
\hline
\end{tabular}




\begin{tabular}{|l|l|l|l|l|}
\hline \multicolumn{1}{|c|}{ Common name } & \multicolumn{1}{|c|}{ Scientific name } & \multicolumn{1}{c|}{ Family } & Years recorded \\
\hline 66 & Square-spot rustic & Xestia xanthographa & Noctuidae & 09 \\
\hline 67 & Antler moth & Cerapteryx graminis & Noctuidae & 09 \\
\hline 68 & Smoky wainscot & Mythimna impura & Noctuidae & 09 \\
\hline 69 & Dark arches & Apamea monoglypha & Noctuidae & 09 \\
\hline 70 & Tawny marbled minor & Oligia latruncula & Noctuidae & 09 \\
\hline 71 & Middle-barred minor & Oligia fasciuncula & Noctuidae & 09 \\
\hline 72 & Tawny marbled or & Oligia strigilis agg. & Oecophoridae & $08,10^{*}$ \\
\hline 73 & Common rustic & Mesapamea secalis & Noctuidae & 09 \\
\hline 74 & Small dotted buff & Photedes minima & Noctuidae & 09 \\
\hline 75 & Burnished brass & Diachrysia chrysitis & Noctuidae & 09 \\
\hline 76 & Beautiful golden & Autographa pulchrina & Noctuidae & 09 \\
\hline 77 & Straw dot & Rivula sericealis & Noctuidae & 09 \\
\hline 78 & The snout & Hypena proboscidalis & Noctuidae & 09 \\
\hline 79 & The fan-foot & Zanclognatha tarsipennalis & Noctuidae & 09 \\
\hline
\end{tabular}

Table 4 Moths recorded at RBGE's Edinburgh Garden, 2008-2011. Primarily based on records made by Duncan Davidson (2008) and Amy Huff (2009). Records made by the author are marked with an asterisk (*). LS: Lawrie Springate; NCR: new vice-county record. None is Listed.

Bees, wasps and ants (Hymenoptera) (2009-2012: Table 5)

At least 48 species of Hymenoptera have been recorded at RBGE since 2009; some records from 2007 and 2008 contain no additional species. They include 3 species of ant, 11 bumblebees, the honey bee, 3 leaf-cutting bees, 20 other solitary bees, 1 cuckoo bee, 2 parasitic wasps, 1 digger wasp, 1 potter wasp, 3 sawflies and 2 social wasps. Five of the solitary bees are on the Scottish Biodiversity List, with two (Colletes daviesanus and Osmia rufa) also on the Edinburgh LBAP notable species list. By far the most noteworthy is the wool carder bee (Fig. 2), a pair of which were recorded on the Rock Garden for several weeks between July and August 2011. This was the first ever record of wool carder bee anywhere in the Lothians, and the first Scottish record outside Dumfries and Galloway, where it is fairly widespread and has been known for over a century. A full account of this discovery will be published elsewhere. At RBGE, the wool carder bees were favouring one of their known food plants, Sempervivum. As RBGE has a 'green roof' on the John Hope Gateway, it remains to be seen whether this will also attract wool carder bees, as 'green roofs' have done elsewhere (Colla et al., 2009; Dunnett et al., 2011), and so encourage RBGE's tiny population to flourish. 


\begin{tabular}{|c|c|c|c|c|c|}
\hline & Species & Family & Group & Listings & $\begin{array}{c}\text { Years } \\
\text { recorded }\end{array}$ \\
\hline 1 & Ichneumon xanthorius & Ichneumonidae & Parasitic wasp & & 10 \\
\hline 2 & Rhyssa persuasoria & Ichneumonidae & Parasitic wasp & & 11 \\
\hline 3 & Mimesa equestris & Sphecidae & Digger wasp & & 09-11 \\
\hline 4 & Ancistrocerus scoticus & Vespidae & Potter wasp & & 09-11 \\
\hline 5 & Dolichovespula sylvestris & Vespidae & Social wasp & & 11 \\
\hline 6 & Vespula vulgaris & Vespidae & Social wasp & & $09-12$ \\
\hline 7 & Nomada marshamella & Anthophoridae & Cuckoo bee & & 10 \\
\hline 8 & Andrena barbilabris & Andrenidae & Solitary bee & & 11 \\
\hline 9 & Andrena bicolor & Andrenidae & Solitary bee & & $09-12$ \\
\hline 10 & Andrena chrysoceles & Andrenidae & Solitary bee & & 09 \\
\hline 11 & Andrena denticulata & Andrenidae & Solitary bee & & 10,11 \\
\hline 12 & Andrena dorsata & Andrenidae & Solitary bee & & 10,11 \\
\hline 13 & Andrena flavipes & Andrenidae & Solitary bee & & 10,11 \\
\hline 14 & Andrena haemorrhoea & Andrenidae & Solitary bee & & 10,11 \\
\hline 15 & Andrena helvola & Andrenidae & Solitary bee & & 10,11 \\
\hline 16 & Andrena nigroaenea & Andrenidae & Solitary bee & & 12 \\
\hline 17 & Andrena scotica & Andrenidae & Solitary bee & & 09,11 \\
\hline 18 & Andrena semilaevis & Andrenidae & Solitary bee & & 11 \\
\hline 19 & Andrena tarsata & Andrenidae & Solitary bee & & 10 \\
\hline 20 & Colletes daviesanus & Colletidae & Solitary bee & $\mathrm{S}, \mathrm{E}$ & 09 \\
\hline 21 & Halictus rubicundus & Halictidae & Solitary bee & & 09,11 \\
\hline 22 & Halictus tumulorum & Halictidae & Solitary bee & & 09,11 \\
\hline 23 & Lasioglossum villosulum & Halictidae & Solitary bee & $\mathrm{S}$ & 09-11 \\
\hline 24 & Lasioglossum calcatum & Halictidae & Solitary bee & & 11 \\
\hline 25 & Anthidium manicatum & Megachilidae & Solitary bee & S & $11 \mathrm{NCR}$ \\
\hline 26 & Megachile centuncularis & Megachilidae & Leaf-cutting bee & & 10 \\
\hline 27 & Megachile ligniseca & Megachilidae & Leaf-cutting bee & & 09 \\
\hline 28 & Megachile willughbiella & Megachilidae & Leaf-cutting bee & & 11 \\
\hline 29 & Osmia rufa & Megachilidae & Solitary bee & $\mathrm{S}, \mathrm{E}$ & 10 \\
\hline 30 & Melitta haemorrhoidalis & Melittidae & Solitary bee & & 09 \\
\hline 31 & Apis mellifera & Apidae & Honey bee & & 09-12 \\
\hline 32 & Bombus bohemicus & Apidae & Bumblebee & & $09,10,12$ \\
\hline 33 & Bombus campestris & Apidae & Bumblebee & & 09-11 \\
\hline
\end{tabular}




\begin{tabular}{|l|l|l|l|l|l|}
\hline \multicolumn{1}{|c|}{ Species } & \multicolumn{1}{|c|}{ Family } & \multicolumn{1}{|c|}{ Group } & Listings & \multicolumn{1}{c|}{$\begin{array}{c}\text { Years } \\
\text { recorded }\end{array}$} \\
\hline 34 & Bombus hortorum & Apidae & Bumblebee & & $09-12$ \\
\hline 35 & Bombus jonellus & Apidae & Bumblebee & & 09,10 \\
\hline 36 & Bombus lapidarius & Apidae & Bumblebee & & $09-12$ \\
\hline 37 & Bombus lucorum & Apidae & Bumblebee & & $09-12$ \\
\hline 38 & Bombus muscorum & Apidae & Bumblebee & S & $09-11$ \\
\hline 39 & Bombus pascuorum & Apidae & Bumblebee & & $09-12$ \\
\hline 40 & Bombus pratorum & Apidae & Bumblebee & & $09-12$ \\
\hline 41 & Bombus sylvestris & Apidae & Bumblebee & & $09-11$ \\
\hline 42 & Bombus terrestris & Apidae & Bumblebee & & $09-12$ \\
\hline 43 & Allantus cinctus & Tenthredinidae & Sawfly & & 09 \\
\hline 44 & Tenthredo arcuata & Tenthredinidae & Sawfly & & 10 \\
\hline 45 & Tenthredo brevicornis & Tenthredinidae & Sawfly & & 11 \\
\hline 46 & Lasius niger & Formicidae & Ant & & $09-11$ \\
\hline 47 & Myrmica ruginodis & Formicidae & Ant & & 10 \\
\hline 48 & Tetramorium caespitum & Formicidae & Ant & & 11 \\
\hline
\end{tabular}

Table 5 Hymenoptera recorded at RBGE's Edinburgh Garden, 2009-2011, with some records from January to March 2012 also included. NCR: new vice-county record. Listings codes as in Table 1.

\section{Hoverflies (2009-2011: Table 6)}

Just over 100 hoverfly species (Diptera: Syrphidae) have been recorded in Edinburgh, as can be found by typing any Edinburgh postcode (between EH1 and EH19) into the Hoverfly Recording Scheme's (HRS) website (Hoverfly Recording Scheme, 2005). At least 52, about half the Edinburgh total, have so far been recorded from the Edinburgh Garden, the vast majority of them, though not all, on the HRS Edinburgh list. All are widespread species such as marmalade hoverfly (Fig. 3) but many have not been recorded in Edinburgh (according to the HRS website) for over ten years, so the city appears to be under-recorded. An example of this under-recording is Volucella bombylans, a distinctive, easily identified bumblebee mimic, which is not listed for Edinburgh on the HRS site but was recorded at RBGE in 2010 and 2011. 


\begin{tabular}{|c|c|c|}
\hline & Species & Years recorded \\
\hline 1 & Anasimyia lineata & 09 \\
\hline 2 & Arctophila superbiens & 09,11 \\
\hline 3 & Baccha elongata & 09,10 \\
\hline 4 & Cheilosia aff. fraterna & 10 \\
\hline 5 & Cheilosia aff. pagana & 11,12 \\
\hline 6 & Cheilosia variabilis & 09 \\
\hline 7 & $\begin{array}{l}\text { Criorhina berberina } \mathrm{f} . \\
\text { oxyacanthae }\end{array}$ & 09 \\
\hline 8 & $\begin{array}{l}\text { Dasysyrphus } \\
\text { albostriatus }\end{array}$ & 09 \\
\hline 9 & $\begin{array}{l}\text { Dasysyrphus cf. } \\
\text { pinastri }\end{array}$ & 09 \\
\hline 10 & $\begin{array}{l}\text { Dasysyrphus cf. } \\
\text { tricinctus }\end{array}$ & 09 \\
\hline 11 & Dasysyrphus venustus & 10,11 \\
\hline 12 & Epistrophe eligans & 09 \\
\hline 13 & Epistrophe grossulariae & 09,10 \\
\hline 14 & Episyrphus balteatus & 09-12 \\
\hline 15 & Eristalis abusivus & 09,11 \\
\hline 16 & Eristalis arbustorum & 09,11 \\
\hline 17 & Eristalis horticola & 09 \\
\hline 18 & Eristalis interruptus & 09,11 \\
\hline 19 & Eristalis intricarius & $09-12$ \\
\hline 20 & Eristalis pertinax & $09-11$ \\
\hline 21 & Eristalis tenax & 09-12 \\
\hline 22 & Eupeodes corollae & $09-11$ \\
\hline 23 & Eupeodes latifasciatus & 09 \\
\hline 24 & Eupeodes luniger & $09-11$ \\
\hline 25 & Helophilus hybridus & $09-11$ \\
\hline 26 & Helophilus pendulus & $09-11$ \\
\hline 27 & Leucozona lucorum & 09,10 \\
\hline
\end{tabular}

\begin{tabular}{|c|c|c|}
\hline & Species & Years recorded \\
\hline 28 & Melangyna cincta & 09 \\
\hline 29 & Melanostoma mellinum & $09-12$ \\
\hline 30 & Melanostoma scalare & 09-11 \\
\hline 31 & Merodon equestris & $\begin{array}{l}\text { 09-11; vars } \\
\text { equestris, } \\
\text { bulborum, } \\
\text { narcissi }\end{array}$ \\
\hline 32 & Myathropa florea & $09-11$ \\
\hline 33 & $\begin{array}{l}\text { Parhelophilus } \\
\text { versicolor }\end{array}$ & 11 \\
\hline 34 & Pipiza noctiluca & 09 \\
\hline 35 & Platycheirus albimanus & $09-11$ \\
\hline 36 & Platycheirus angustatus & 10 \\
\hline 37 & Platycheirus peltatus & 11 \\
\hline 38 & Platycheirus scambus & 09 \\
\hline 39 & Platycheirus scutatus & 10,11 \\
\hline 40 & Scaeva pyrastri & 11 \\
\hline 41 & Sericomyia silentis & 10 \\
\hline 42 & Sphaerophoria fatarum & 09,10 \\
\hline 43 & $\begin{array}{l}\text { Sphaerophoria } \\
\text { interrupta }\end{array}$ & 11 \\
\hline 44 & $\begin{array}{l}\text { Sphaerophoria } \\
\text { philanthus }\end{array}$ & 09 \\
\hline 45 & Sphaerophoria scripta & 09-11 \\
\hline 46 & Syritta pipiens & 09 \\
\hline 47 & Syrphus ribesii & 09-11 \\
\hline 48 & Syrphus torvus & 10,11 \\
\hline 49 & Syrphus vitripennis & 09-11 \\
\hline 50 & Volucella bombylans & $\begin{array}{l}10 \text { (f. plumata), } \\
11\end{array}$ \\
\hline 51 & Volucella pellucens & 09-11 \\
\hline 52 & Xylota segnis & 10 \\
\hline
\end{tabular}

Table 6 Hoverflies (Syrphidae) recorded at RBGE's Edinburgh Garden, 2009-2011 (including first records of 2012 season). None is Listed. 


\section{Odonata (2005-2011: Table 7)}

So far three dragonflies and four damselflies have been recorded. The most commonly seen are azure damselfly (an annual species, particularly favouring the small pond in the Scottish Heath Garden), and common hawker. The other five species listed in Table 7 are recorded less frequently. In 2011 a decision was taken to manage the Scottish Heath Garden pond to encourage its use by Odonata.

\begin{tabular}{|c|l|l|l|l|}
\hline \multicolumn{1}{|c|}{ Common name } & \multicolumn{1}{|c|}{ Scientific name } & \multicolumn{1}{c|}{ Family } & \multicolumn{1}{c|}{ Years recorded } \\
\hline 1 & Azure damselfly & Coenagrion puella & Coenagrionidae & pre-2008, 08-11 \\
\hline 2 & Blue-tailed damselfly & Ischnura elegans & Coenagrionidae & pre-2008, 11 \\
\hline 3 & Common blue damselfly & Enallagma cyathigerum & Coenagrionidae & pre-2008 \\
\hline 4 & Large red damselfly & Pyrrhosoma nymphula & Coenagrionidae & pre-2008, 09 \\
\hline 5 & Common hawker & Aeshna juncea & Aeshnidae & pre-2008, 08-10 \\
\hline 6 & Black darter & Sympetrum danae & Libellulidae & pre-2008 \\
\hline 7 & Common darter & Sympetrum striolatum & Libellulidae & pre-2008 \\
\hline
\end{tabular}

Table 7 Odonata (dragonflies and damselflies) recorded at RBGE's Edinburgh Garden, 2008-2011. None is Listed.

\section{Other insects (Table 8)}

The 11 beetle species so far recorded at RBGE include 3 species of ladybird (two-spot, seven-spot and pine) and 8 others such as green leaf weevil (Fig. 4). The alien harlequin ladybird, Harmonia axyridis, does not yet appear to have been found at RBGE - a photograph on Flickr taken in RBGE (DFG Photography, 2011) captioned as the species instead shows a melanistic two-spot ladybird, a variant I have seen myself in RBGE alongside the typical red form of that species.

Twenty-six fly species (including leaf-miners) have so far been recorded at RBGE. They include the rather scarce greater bee-fly, which can be seen hovering in front of plants in the Rock Garden in April and May; the St Mark's fly, vast hordes of which thronged the Garden in 2011; and some very attractive small 'picture-winged' fruit flies such as Campiglossa misella and Euleia heraclei. The nine bugs that have been recorded include four shield bugs, the alder spittlebug, the ubiquitous meadow froghopper and the pond skater. One lacewing has been recorded. Unusually this was the black lacewing (Fig. 5), recorded in June 2011 and only the fifth Scottish record of the species. A full report of this finding will be published elsewhere. Another notable discovery was of the barkfly Trichopsocus brincki on Pinus strobus in 2007 by Bob Saville (Saville et al., 2008) - the first Scottish record of this nationally scarce species. 


\begin{tabular}{|c|c|c|c|c|c|}
\hline & Common name & Scientific name & Family & Order & $\begin{array}{l}\text { Years } \\
\text { recorded }\end{array}$ \\
\hline 1 & a soldier beetle & Cantharis livida & Cantharidae & $\mathrm{Col}$ & 10 \\
\hline 2 & a soldier beetle & Cantharis nigricans & Cantharidae & $\mathrm{Col}$ & 11 \\
\hline 3 & $\begin{array}{l}\text { Common red soldier } \\
\text { beetle }\end{array}$ & Rhagonycha fulva & Cantharidae & $\mathrm{Col}$ & 09-11 \\
\hline 4 & a leaf beetle & Phratora cf. laticollis & Chrysomelidae & $\mathrm{Col}$ & 11 \\
\hline 5 & Two-spot ladybird & Adalia bipunctata & Coccinellidae & $\mathrm{Col}$ & 11 \\
\hline 6 & Seven-spot ladybird & Coccinella septempunctata & Coccinellidae & $\mathrm{Col}$ & 09-11 \\
\hline 7 & Pine ladybird & $\begin{array}{l}\text { Exochomus } \\
\text { quadripustulatus }\end{array}$ & Coccinellidae & $\mathrm{Col}$ & 10,11 \\
\hline 8 & Green leaf weevil & Phyllobius maculicornis & Curculionidae & $\mathrm{Col}$ & 11 \\
\hline 9 & Common pollen beetle & Meligethes aeneus & Nitidulidae & Col & 10 \\
\hline 10 & Bee beetle & Trichius fasciatus & Scarabaeidae & Col & 09 \\
\hline 11 & Rose chafer & Cetonia aurata & Scarabaeidae & $\mathrm{Col}$ & 10 \\
\hline 12 & a leaf-mining fly & Aulagromyza cornigera & Agromyzidae & Dip & 11 \\
\hline 13 & a leaf-mining fly & Phytomyza heracleana & Agromyzidae & Dip & 11 \\
\hline 14 & a leaf-mining fly & Phytomyza ilicis & Agromyzidae & Dip & 11 \\
\hline 15 & a leaf-mining fly & $\begin{array}{l}\text { Phytomyza pastinacae / } \\
\text { sphondylii }\end{array}$ & Agromyzidae & Dip & 11 \\
\hline 16 & St Mark's fly & Bibio marci & Bibionidae & Dip & 10,11 \\
\hline 17 & Greater bee-fly & Bombylius major & Bombyliidae & Dip & $\begin{array}{l}10,11, \\
12\end{array}$ \\
\hline 18 & Bluebottle & Calliphora vomitoria & Calliphoridae & Dip & 10,11 \\
\hline 19 & Greenbottle & Lucilia sp. & Calliphoridae & Dip & $09-11$ \\
\hline 20 & a gall-forming fly & Anisostephus betulinus & Cecidomyiidae & Dip & 11 \\
\hline 21 & a conopid fly & Conops quadrifasciatus & Conopidae & Dip & 10 \\
\hline 22 & a fly & Gymnopternus cf. cupreus & Dolichopodidae & Dip & 11 \\
\hline 23 & a fly & Coenosia agromyzina & Muscidae & Dip & 10 \\
\hline 24 & a fly & Eudasyphora cyanicolor & Muscidae & Dip & 11 \\
\hline 25 & House fly & Musca domestica & Muscidae & Dip & $09-12$ \\
\hline 26 & a fly & Mydaea cf. affinis & Muscidae & Dip & 11 \\
\hline 27 & a fly & Polietes lardarius & Muscidae & Dip & 09 \\
\hline 28 & a flutter-wing fly & $\begin{array}{l}\text { Palloptera } \\
\text { quinquemaculata }\end{array}$ & Pallopteridae & Dip & 11 \\
\hline 29 & a flesh-fly & Sarcophaga sp. & Sarcophagidae & Dip & 11 \\
\hline 30 & a midge & Reichertella geniculata & Scatopsidae & Dip & 11 \\
\hline
\end{tabular}




\begin{tabular}{|l|l|l|l|l|l|}
\hline \multicolumn{1}{|c|}{ Common name } & \multicolumn{1}{|c|}{ Scientific name } & \multicolumn{1}{|c|}{ Family } & Order & \multicolumn{1}{c|}{$\begin{array}{l}\text { Years } \\
\text { recorded }\end{array}$} \\
\hline 31 & $\begin{array}{l}\text { Scarce orange } \\
\text { legionnaire }\end{array}$ & Beris clavipes & Stratiomyidae & Dip & 11 \\
\hline 32 & a soldier fly & Chloromyia formosa & Stratiomyidae & Dip & 11 \\
\hline 33 & a parasitic fly & Tachina fera & Tachinidae & Dip & $09-11$ \\
\hline 34 & a fruit fly & Campiglossa misella & Tephritidae & Dip & 10 \\
\hline 35 & a fruit fly & Euleia heraclei & Tephritidae & Dip & 11 \\
\hline 36 & Cranefly sp. & Tipula sp. & Tipulidae & Dip & 10 \\
\hline 37 & Winter gnat & Trichocera sp. & Trichoceridae & Dip & 11,12 \\
\hline 38 & Hawthorn shieldbug & $\begin{array}{l}\text { Acanthosoma } \\
\text { haemorrhoidale }\end{array}$ & Acanthosomatidae & Hem & 11 \\
\hline 39 & Birch shieldbug & Elasmostethus interstinctus & Acanthosomatidae & Hem & 10,11 \\
\hline 40 & Alder spittlebug & Aphrophora alni & Aphrophoridae & Hem & 11 \\
\hline 41 & Meadow froghopper & Philaenus spumarius & Aphrophoridae & Hem & 11 \\
\hline 42 & Pond skater & Gerris lacustris & Gerridae & Hem & $09-11$ \\
\hline 43 & Black-kneed capsid & $\begin{array}{l}\text { Blepharidopterus } \\
\text { angulatus }\end{array}$ & Miridae & Hem & 11 \\
\hline 44 & a mirid bug & Dolycoris baccarum & Pentatomidae & Hem & 11 \\
\hline 45 & Sloe bug & Pentatoma rufipes & Pentatomidae & Hem & 10,11 \\
\hline 46 & Forest bug & Trichopsocus brincki & Trichopsocidae & Pso & 07 \\
\hline 48 & Black lacewing & Chrysopidae & Neu & 11 \\
\hline
\end{tabular}

Table 8 Miscellaneous other insects recorded at RBGE's Edinburgh Garden, 2009-2011. None is Listed. Orders: Col: Coleoptera; Dip: Diptera; Hem: Hemiptera; Neu: Neuroptera; Pso: Psocoptera.

\section{Spiders and gall mites (2011-)}

Recording of these has only just begun but already at least eight gall-forming mites and five spiders have been recorded. The latter include the bright green cucumber spider (Araniella cucurbitina) and the zebra spider (Salticus scenicus), which is a jumping species. The exotic jumping species Hasarius adansoni (Fig. 6) was re-found in December 2011 in one of the glasshouses. The same species had been recorded from RBGE's glasshouses by W. Edgar Evans in 1904 (Carpenter \& Evans, 1906) and by Robert Godfrey in 1905 (Pickard-Cambridge, 1906). 


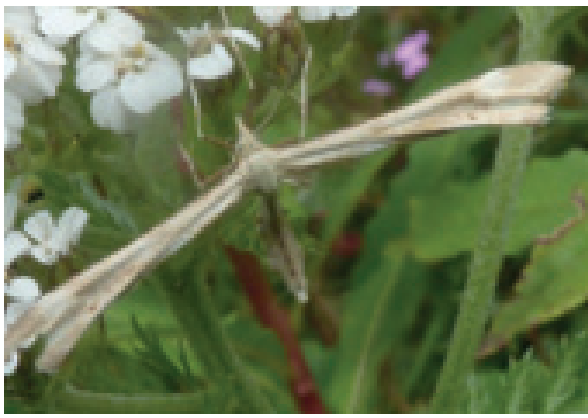

Fig. 1 Platyptilia pallidactyla (yarrow plume), 21 July 2011. Photo: Robert Mill.

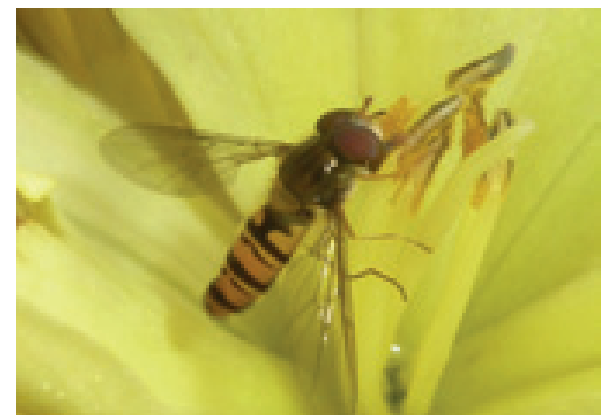

Fig. 3 Episyrphus balteatus (marmalade hoverfly), 3 August 2011. Photo: Robert Mill.

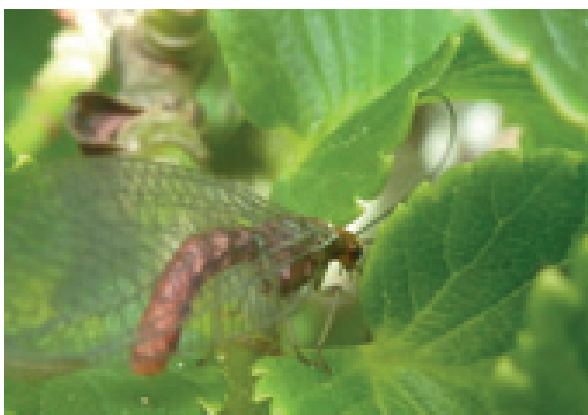

Fig. 5 Nothochrysa capitata (black lacewing), 22 June 2011. Fifth record for Scotland. Photo: Robert Mill.

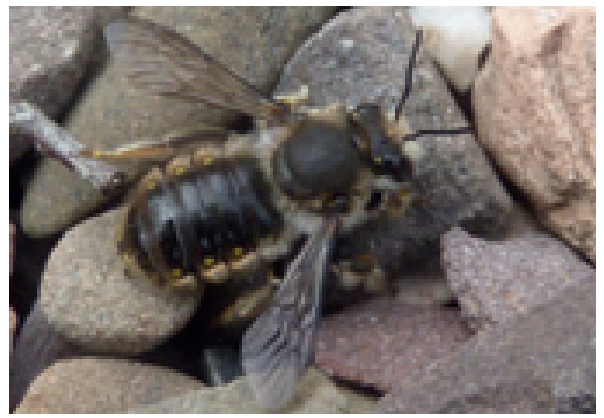

Fig. 2 Anthidium manicatum (wool carder bee), 3 August 2011. First record for the Lothians. Photo: Robert Mill.

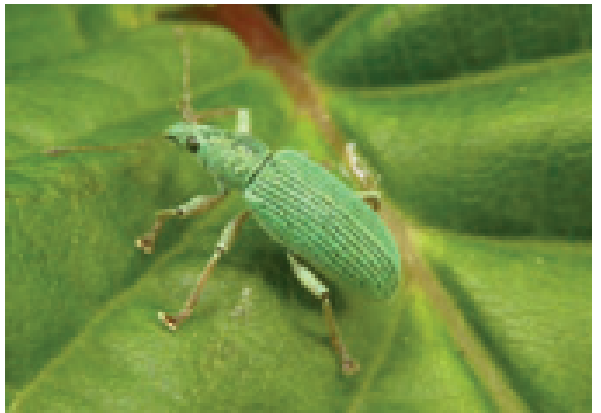

Fig. 4 Phyllobius maculicornis (green leaf weevil), 30 May 2011. Photo: Robert Mill.

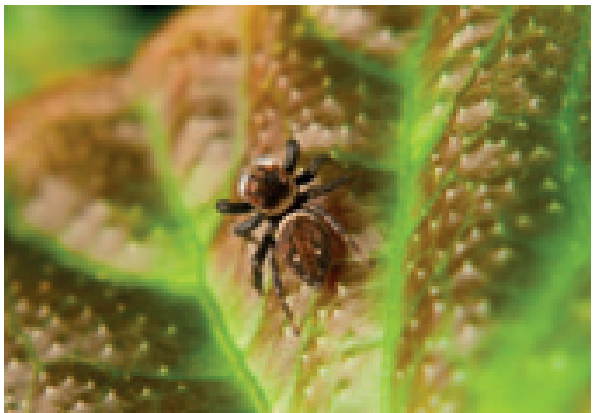

Fig. 6 Hasarius adansoni, on a Begonia leaf, December 2011. Re-discovered in the glasshouses after 108 years. Photo: Lynsey Wilson. 
Zooplankton (2010)

Zooplankton were sampled by Ian Duggan in two of the outdoor ponds and the ponds in the Plants and People and Rainforest Riches glasshouses. Twelve rotifers, five cladocerans and one copepod, together with rotifer and copepod larval stages, were identified.

\section{CONCLUSIONS}

Recording the wildlife of RBGE's Edinburgh Garden has yielded much new information, including several new records for the Lothians and a major range extension within Scotland for wool carder bee. Much remains to be done, particularly in less well-known groups that are difficult to identify and where there are few experts. Jennifer Owen recorded 2,199 wildlife species in her Leicestershire garden over 30 years, including 1,997 insects and 138 other invertebrates (Owen, 2010); compared with that total the Edinburgh figure, for a much larger garden, is very small but probably reflects both habitat differences and under- or non-recording of many groups. The RBGE Biodiversity Group is planning to hold a 'BioBlitz' event in 2013 that should address some of these issues by gathering together a wide range of experts and engaging the public. A BioBlitz is the intensive surveying of the biodiversity of an area over a 24-hour period. It is also proposed to compile biodiversity inventories for the other three botanic gardens managed by RBGE at Benmore, Dawyck and Logan. This will be a difficult task because of the lack of experts on the ground. Organising BioBlitzes at each Garden over a period of years could, however, greatly assist in increasing our as yet scant knowledge of the wildlife of those three places, currently limited to lists of birds, mammals and amphibians plus a short moth list for Logan.

\section{ACKNOWLEDGEMENTS}

I thank Duncan Davidson and Amy Huff for moth trapping records, Amy Huff also for some records of leaf-miners and galling insects and mites, Hugh and Laura Coventry for pre-2007 records of butterflies, dragonflies and damselflies and their continued interest in recording wildlife at RBGE, Ian Duggan for records of zooplankton in 2010, Max Coleman for facilitating the display of daily wildlife sightings at the John Hope Gateway, Lynsey Wilson for the photograph of Hasarius adansoni and the many RBGE staff who pass on their own wildlife records to me and thus enhance our knowledge of the wildlife of the Edinburgh Garden.

\section{REFERENCES}

BLAKE, K. (2005). Production of the list of species and habitats considered to be of principal importance for the purpose of conservation of biodiversity in Scotland (The Scottish 
Biodiversity List). Part 2 - Technical Report. Final Report. Scott Wilson, Edinburgh. $112 \mathrm{pp}$.

BRITISH TRUST FOR ORNITHOLOGY (2009). Birds of Conservation Concern 3. Available online: http://www.bto.org/sites/default/files/u37/downloads/recording/bocc3.pdf (accessed March 2012).

BUTTERFLY CONSERVATION SCOTLAND (2008). East Scotland butterflies. Butterfly Conservation Scotland Summer e-newsletter 2008, 4. Available online: http://www.butterflyconservation.org/uploads/Summer\%20e-news\%202008.pdf (accessed March 2012).

CARPENTER, G.H. \& EVANS, W. (1906). Further additions to the list of spiders from the Edinburgh district. Proceedings of the Royal Physical Society of Edinburgh, 16, 27-34.

CLEAR, S. (2005). Factors affecting the distribution of Pipistrellus pipistrellus and Pipistrellus pygmaeus in the Lothians region, Scotland. BaTML Publications, 2, 23-33.

COLLA, S.R., WILLIS, E. \& PACKER, L. (2009). Can green roofs provide habitat for urban bees (Hymenoptera: Apidae)? Cities and the Environment, 2(1), article 4.

DFG PHOTOGRAPHY (2011). Harlequin ladybird. Available online: http://www.flickr.com/ photos/lothianlife/5605845930/ (accessed March 2012).

DUNCAN, J. (1999). Do you still need - Hawfinch? Birding Scotland, 2(1), 29-30.

DUNNETT, N., GEDGE, D., LITTLE, J. \& SNODGRASS, E.C. (2011). Small green roofs: lowtech options for greener living. Timber Press, Portland, OR \& London.

EDINBURGH BIODIVERSITY PARTNERSHIP (2010a). Edinburgh Local Biodiversity Action Plan 2010-2015. Available online: http://www.edinburgh.gov.uk/downloads/file/1956/ edinburgh_biodiversity_action_plan (accessed March 2012).

EDINBURGH BIODIVERSITY PARTNERSHIP (2010b). Notable Species List for Edinburgh. Available online: http://www.edinburgh.gov.uk/downloads/file/444/notable_species_list (accessed March 2012).

FOX, R., WARREN, M.S. \& BRERETON, T.M. (2010). The Butterfly Red List for Great Britain. Species Status, 12, 1-32.

HARRIS, S., MORRIS, P., WRAY, S. \& YALDEN, D. (1995). A review of British mammals: population estimates and conservation status of British mammals other than cetaceans. JNCC, Peterborough. Available online: http://jncc.defra.gov.uk/pdf/pub03_ areviewofbritishmammalsall.pdf (accessed March 2012).

HOVERFLY RECORDING SCHEME (2005). Available online: http://www.hoverfly.org.uk/ portal.php (accessed March 2012).

MCBEATH, R. (1989). (32) Royal Botanic Garden, Edinburgh (NT244753). In: ANDREWS, I.J., Birdwatching sites in the Lothians. Available online: http://www.andrewsi.freeserve. co.uk/lothian-sites.htm (accessed March 2012).

MIDDLETON, N.E. (2006). A study of the emission of social calls by Pipistrellus spp. within central Scotland; including a description of their typical social call structure. BaTML Publications, 3, 23-28.

MILL, R.R. (2012). List of notable wildlife species at RBGE Inverleith and Benmore. RBGE, unpublished internal document.

OWEN, J. (2010). Wildlife of a Garden. Royal Horticultural Society, Peterborough. 
PICKARD-CAMBRIDGE, O. (1906). On some new and rare British Arachnida. Proceedings of the Dorset Natural History and Antiquarian Field Club 27, 72-92.

PLANT, C.W. (1994). Provisional atlas of the lacewings and allied insects (Neuroptera, Megaloptera, Raphidioptera and Mecoptera) of Britain and Ireland. Monks Wood, Biological Records Centre, JNCC.

ROYAL BOTANIC GARDEN EDINBURGH (2011). Environmental Policy. Royal Botanic Garden Edinburgh.

SAVILLE, R.E.S., ALEXANDER, K.N.A. \& OLDFIELD, M. (2008). Notable British barkfly (Psocoptera) observations in 2007. Entomologist's Record, 120, 19-20.

SCOTTISH BIODIVERSITY FORUM/SCOTTISH EXECUTIVE (2004). Scotland's biodiversity: it's in your hands. A strategy for the conservation and enhancement of biodiversity in Scotland. Scottish Executive, Edinburgh.

SCOTTISH NATURAL HERITAGE (2010). Scottish Biodiversity List. Available online: http://www.snh.gov.uk/planning-and-development/advice-for-planners-and-developers/ biodiversity/ (accessed March 2012).

SCOTTISH PARLIAMENT (2004). Nature Conservation (Scotland) Act 2004 (asp 6). Stationery Office, Edinburgh.

SCOTTISH PARLIAMENT (2011a). Wildlife and Natural Environment (Scotland) Act 2011 (asp 6). Stationery Office, Edinburgh.

SCOTTISH PARLIAMENT (2011b). The Wildlife and Natural Environment (Scotland) Act 2011 (Commencement No. 2) Order 2011. Scottish Statutory Instrument 2011 No. 433 (C. 37). Stationery Office, Edinburgh.

SOMERSET MOTH GROUP (2012). Standard Weeks. Available online: http://www. somersetmothgroup.org.uk/portal/p/Standard+Weeks (accessed March 2012).

SUTCLIFFE, R. (2009). Recent changes in the distribution of some Scottish butterflies and the arrival of new species in Scotland. The Glasgow Naturalist, 25(2), 5-12. 